\title{
Short-distance constraints for HLbL in muon g-2
}

\author{
Johan Bijnens ${ }^{1, *}$, Nils Hermansson-Truedsson ${ }^{2, * *}$, and Antonio Rodríguez-Sánchez ${ }^{3, * * *}$ \\ ${ }^{1}$ Department of Astronomy and Theoretical Physics, Lund University, Box 43, SE221-00 Lund, Sweden \\ ${ }^{2}$ Albert Einstein Center for Fundamental Physics, Institute for Theoretical Physics, Universität Bern, \\ Sidlerstrasse 5, CH-3012 Bern, Switzerland \\ ${ }^{3}$ Université Paris-Saclay, CNRS/IN2P3, IJCLab, 91405 Orsay, France
}

\begin{abstract}
Model-independent short-distance constraints allow for a reduction of theoretical uncertainties associated to the analytic evaluation of Hadronic Light-by-Light contributions to the muon g-2. In this talk we focus on the region where the three loop virtualities are large. Even when the fourth photon leg is soft, we show how a precise Operator Product Expansion can be applied in that region. The leading contribution is found to be given by the quark loop, while the evaluation of both gluonic and power corrections show how the expansion is well behaved at relatively low energies, where significant contributions to the muon $\mathrm{g}-2$ remain. Numerical values for them are also presented.
\end{abstract}

\section{Introduction}

A new measurement of the anomalous magnetic moment of the muon, coming from the FNAL Muon g-2 Experiment, was released this year [1], updating the combined experimental average to

$$
a_{\mu}^{\exp }=116592061(41) \cdot 10^{-11},
$$

and confirming the tension with the SM prediction of Ref. [2],

$$
a_{\mu}^{\mathrm{SM}}=116591810(43) \cdot 10^{-11} .
$$

Such a remarkable theoretical precision is in part a consequence of the strong dominance of pure QED contributions, whose theory uncertainties are completely negligible compared to the experimental result. Electroweak corrections are very small and well known too, so they cannot, within the SM, account for such a tension. A discrepancy of $\sim 2.5 \cdot 10^{-8}$ with perfectly controlled corrections from strong interactions would immediately translate into evidence of BSM physics. In order to achieve such a control, strong efforts are being made by both the data-driven and lattice approaches in the two relevant contributions: Hadronic Vacuum Polarization (HVP) and Hadronic Light-by-Light contributions (HLbL). The focus of our work, [3-5], has been oriented to improve the data-driven evaluation of the latter.

\section{Hadronic Light-by-Light contributions to the muon g-2}

The Hadronic Light-by-Light contribution to g-2 is depicted in Fig. 1. The key piece for its calculation is the corresponding HLbL tensor, defined as

\footnotetext{
*e-mail: johan.bijnens@thep.lu.se

**e-mail: nils@itp.unibe.ch

*** Speaker, e-mail: arodriguez@ijclab.in2p3.fr
} 


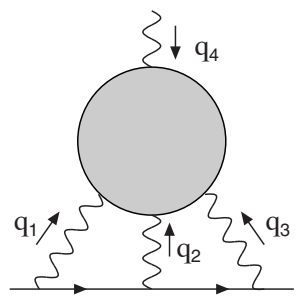

Figure 1. HLbL contribution to g-2.

$$
\Pi^{\mu_{1} \mu_{2} \mu_{3} \mu_{4}} \equiv-i \int \frac{d^{4} q_{4}}{(2 \pi)^{4}}\left(\prod_{i=1}^{4} \int d^{4} x_{i} e^{-i q_{i} x_{i}}\right)\left\langle 0\left|T\left(\prod_{j=1}^{4} J^{\mu_{j}}\left(x_{j}\right)\right)\right| 0\right\rangle,
$$

where $J^{\mu}(x)=\bar{q}(x) Q_{q} \gamma^{\mu} q(x)$ is the electromagnetic quark current. The correponding g-2 number is obtained when convoluting

$$
\lim _{q_{4} \rightarrow 0} \frac{\partial \Pi^{\mu_{1} \mu_{2} \mu_{3} v_{4}}}{\partial q_{4}^{\mu_{4}}}
$$

with the other two loops $[6,7]$

$$
a_{\mu}^{\mathrm{HLbL}}=\frac{2 \alpha^{3}}{3 \pi^{2}} \int_{0}^{\infty} d Q_{1} \int_{0}^{\infty} d Q_{2} \int_{-1}^{1} d \tau \sqrt{1-\tau^{2}} Q_{1}^{3} Q_{2}^{3} \sum_{i=1}^{12} T_{i}\left(Q_{1}, Q_{2}, \tau\right) \bar{\Pi}_{i}\left(Q_{1}, Q_{2}, \tau\right) .
$$

The $\bar{\Pi}_{i}$ functions only depend on the five independent scalar functions of the Lorentz decomposition of Eq. (4) [5]. The main difficulty in the evaluation of this contribution is finding those functions. The weights $T_{i}$ enhance the low-energy contributions, since the scale of the problem is the muon mass. Perturbative QCD cannot be used for the tensor of Eq. (3) at that energy and non-perturbative methods have to be applied. QCD-inspired evaluations of it gave a first assessment of this contribution [8-14], but a more model-independent evaluation of it was desirable. That possibility arrived with the data-driven approach of Ref. [6], where a model-independent way of evaluating the different leading long-distance contributions was assessed. ${ }^{1}$

While systematic improvements on them are possible, the residual contributions from medium and short distances are more challenging. One of the expected contributions at short distances was the quark loop, but the low-energy scale of the muon mass and the static g-2 photon requires some lower cut-off, since the logarithmic mass divergences associated to the light-by-light contributions do not make much sense for light-quarks. There are no physical hadronic states with such small masses. A constituent quark mass was typically used as a regulator (see for example Ref. [9]). However, in the context of a data-driven evaluation, such a model-dependent solution was not satisfactory. Our work has been focused on the interplay of the short-distance contributions to g-2 within QCD, aiming to see whether and how the quark loop is the leading term of any asymptotic expansion in the g-2 kinematics.

\footnotetext{
${ }^{1}$ Very recently lattice methods have achieved a similar precision [15]. The very good agreement between two largely model-independent and completely different approaches reinforces the idea that a miss-evaluation of HLbL cannot be behind the current tension in muon $\mathrm{g}-2$.
} 


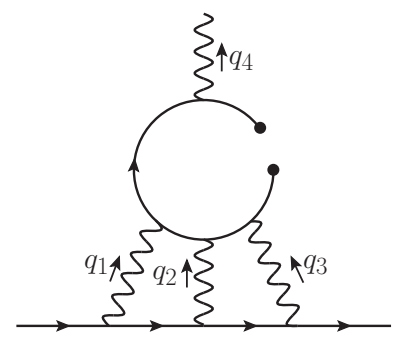

Figure 2. Quark condensate contribution to HLbL. In the static $q_{4} \rightarrow 0$ limit the quark propagator on the left diverges.

\section{The Operator Product Expansion in the QCD vacuum}

Two-point correlation functions of quark currents $J_{1}$ and $J_{2}$,

$$
\Pi(q)=\int d^{4} x e^{-i q x}\left\langle 0\left|T\left(J_{1}(x) J_{2}(0)\right)\right| 0\right\rangle,
$$

are described at large Euclidean Momenta by its Operator Product Expansion (OPE) in the presence of the QCD vacuum [16],

$$
\Pi \approx \sum_{i, D} \frac{c_{i, D} O_{i, D}}{Q^{D}},
$$

where $D$ is the dimension of the associated operator $O_{i, D}, c_{i, D}$ are $c$-numbers, the Wilson coefficients, and $Q^{2}=-q^{2}$. Any possible operator with the same quantum numbers as the QCD vacuum can give a nonzero contribution, starting by unity, which provides the perturbative series, and supplemented by operators with quarks and gluons such as the scalar current $\bar{q} q$, which gives rise to the well-known quark condensate, $\langle\bar{q} q\rangle$. A known limitation of this description for some of these expansions is in the separation of the tail of the perturbative expansion, of asymptotic nature, and the effect of vacuum condensates. Some possible prescriptions on how to separate those effects can be found in the literature (e.g. see [17]). The same OPE can be applied to correlation functions with a higher number of external legs. A detailed study of the OPE of three-point correlation functions can be found in Ref. [18].

We then have an asymptotic expansion describing the behaviour of the HLbL tensor of Eq. (3) at large Euclidean momenta, whose leading contribution is clearly given by the quark loop. Its applicability to the muon g-2 kinematics is, however, not obvious even when we explore the region with three large (Euclidean, $Q_{1,2,3}$ ) loop momenta. This is because the fourth (g-2) momentum is defined in the static $q_{4} \rightarrow 0$ limit, where the use of such a vacuum OPE is not justified. Indeed, an explicit exploration shows how the first dimensional correction, the one associated to the quark condensate, presents divergences in that limit from the diagram shown in Fig. 2 [3]. This observation may cast some doubts on the validity of the quark loop as the leading term of the (subleading) short-distance contributions to HLbL g-2.

\section{The Operator Product Expansion in the QCD vacuum with an external background photon field}

Completely analogous problems for extrapolating the QCD vacuum OPE to the staticmomentum regime were found when trying to describe the nucleon magnetic moments 

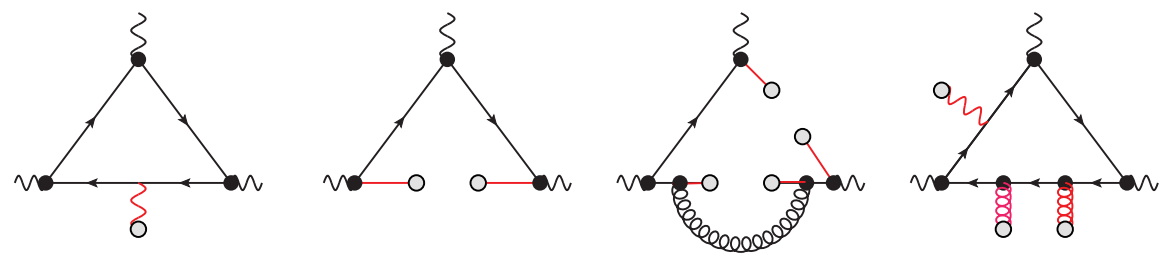

Figure 3. Topologies giving the different contributions to the studied OPE.

through sum rules. A very elegant solution was presented in Refs. [19, 20], and some applications to other muon contributions can actually be found in the literature, e.g. [21]. The essential point is that the OPE should be performed keeping the external g-2 photon as an explicit external (background) state. This is applying the OPE to the (equivalent) tensor

$$
\Pi^{\mu_{1} \mu_{2} \mu_{3}}\left(q_{1}, q_{2}\right) \equiv-\frac{1}{e} \int \frac{d^{4} q_{3}}{(2 \pi)^{4}}\left(\prod_{i=1}^{3} \int d^{4} x_{i} e^{-i q_{i} x_{i}}\right)\left\langle 0\left|T\left(\prod_{j=1}^{3} J^{\mu_{j}}\left(x_{j}\right)\right)\right| \gamma_{E}\left(q_{4}\right)\right\rangle,
$$

where the $\gamma_{E}\left(q_{4}\right)$ is the external static photon. Essentially, from such an OPE one needs to keep not those local operators with the same quantum numbers as the QCD vacuum, but those operators with the same quantum numbers as the external g-2 photon, $F_{\mu \nu}$,

$$
Q_{1, \mu \nu} \equiv e e_{q} F_{\mu \nu}, \quad Q_{2, \mu \nu} \equiv \bar{q} \sigma_{\mu \nu} q \quad, \ldots
$$

The leading contribution is associated to the lowest dimensional operator, $Q_{1}$. This is represented in the first diagram from the left of Fig. 3. We showed in Ref. [3] how, up to gluonic corrections, it corresponds to the massless quark loop, which becomes the leading term in a well-defined expansion.

\section{Power corrections}

Some remarkable things occur when trying to assess the leading quark mass corrections. One would naively expect that the perturbative mass correction should be suppressed by a factor

$$
\frac{\sum_{q} m_{q}^{2} e_{q}^{4}}{Q^{2} \sum_{q} e_{q}^{4}}
$$

which at $Q_{1} \approx Q_{2} \approx Q_{3}=1 \mathrm{GeV}$ gives, taking $m_{u}=m_{d}=5 \mathrm{MeV}$ and $m_{s}=100 \mathrm{MeV}$, $\sim 6 \cdot 10^{-4}$. An explicit computation gives instead $\sim 5 \cdot 10^{-3}$. A careful inspection of the quark loop mass corrections shows that they scale as [4]

$$
\frac{\sum_{q} m_{q}^{2} e_{q}^{4} \ln \frac{Q^{2}}{m_{q}^{2}}}{Q^{2} \sum_{q} e_{q}^{4}}
$$

which explains the enhancement. However, perturbative QCD series with such mass singularities diverge and then such a enhancement is, in principle, unphysical. The standard operator renormalization allows for a complete separation of the contributions in short-distance pieces $c_{O}(\mu)$ and long-distance matrix elements $\langle O(\mu)\rangle$ that must be evaluated nonperturbatively [22]. In the OPE in the vacuum there are no (relative to the leading order pieces) $D=2$ 
operators to regularize $m_{q}^{2} \ln \frac{Q^{2}}{m_{q}^{2}}$ divergences, but they do not appear when dealing with physical observables $[22,23]$. They start at $m_{q}^{4} \ln \frac{Q^{2}}{m_{q}^{2}}$ and are regularized by the quark and gluon condensates. In this case they are regularized by the QED mixing of the tensor current $\bar{q} \sigma^{\mu v} q$ with the leading $F_{\mu \nu}$ contribution [4]. The long-distance quark mass dependence gets absorbed into the mass corrections of the nonperturbative matrix element $\left\langle 0\left|\bar{q} \sigma^{\mu v} q(\mu)\right| \gamma_{E}\left(q_{4}\right)\right\rangle$.

However, in the neighbourhood of the $m_{q} \rightarrow 0$ limit, the leading quark mass correction is not quadratic but linear in the mass and is given by the contribution of the second diagram in Fig 3 to the tensor current, which has a non-zero expectation value in the presence of an external background photon even in the strict chiral limit. This effect of spontaneous chiral symmetry breaking is known as the magnetic susceptibility of the QCD vacuum and is trivially related to the zero momentum limit of $\Pi_{V T}(q)$. The explicit results associated to these contributions, which scale as

$$
\frac{(4 \pi)^{2} m_{q} X_{q}}{Q^{2}} \sim \frac{\Lambda_{\chi}^{2}}{M_{\rho}^{2}} \frac{M_{\pi(K)}^{2}}{Q^{2}},
$$

can be found in Refs. [3, 4] and may be used as short-distance constraints for possible evaluations of mass corrections to chiral limit evaluations of HLbL.

Beyond the chiral limit, the leading power corrections are suppressed by four powers of the large loop momenta. The associated topologies are the ones of Fig. 3. Details in the computation and explicit expressions for them can be found in Ref. [4]. We have checked that, as far as one is above $1 \mathrm{GeV}$, the power corrections are typically suppressed by at least two orders of magnitude with respect to the leading massless quark loop and can be safely neglected.

\section{The two-loop corrections}

In principle the gluonic corrections to the HLbL tensor contain many different two-loop diagrams which depend on where to connect the gluon lines, also with respect to the soft static photon. However, a simplification occurs when realizing that the color structure carried by the gluon can be factored out and it can be regarded as an extra "photon". Then, before contracting the gluon propagator and setting the external g-2 momentum to zero, we simply have a fully symmetric sum of hexagons. It is then a natural step to take advantage of this strong symmetry to eventually break it with both the soft-photon and the second (gluon-propagator) loop. This very simple set-up leads to a very large number of two-loop integrals. But then we can use general projectors $P_{\mu_{1} \mu_{2} \mu_{3} \mu_{4} v_{4}}^{\tilde{n}_{u}}$ to the five independent scalar functions $\tilde{\Pi}_{i}$ of the HLbL $\mathrm{g}-2$ tensor. One finds [5]

$$
\begin{aligned}
\tilde{\Pi}_{i} & =P_{\mu_{1} \mu_{2} \mu_{3} \mu_{4} v_{4}}^{\tilde{q}_{q_{4} \rightarrow 0}} \lim \frac{\partial \Pi^{\mu_{1} \mu_{2} \mu_{3} \nu_{4}}}{\partial q_{4}^{\mu_{4}}} \\
& =-\frac{\left(N_{c}^{2}-1\right) g_{s}^{2} e_{q}^{4}}{4} \int \frac{d^{4} q_{5}}{(2 \pi)^{4}} \frac{g_{\mu_{5} \mu_{6}}}{q_{5}^{2}} \lim _{\substack{q_{4} \rightarrow 0 \\
q_{6} \rightarrow-q_{5}}} P_{\mu_{1} \mu_{2} \mu_{3} \mu_{4} v_{4}}^{\tilde{\Pi}_{i}} \frac{\partial}{\partial q_{4}^{v_{4}}} H^{\mu_{1} \mu_{2} \mu_{3} \mu_{4} \mu_{5} \mu_{6}},
\end{aligned}
$$

where

$$
\begin{aligned}
H^{\mu_{1} \mu_{2} \mu_{3} \mu_{4} \mu_{5} \mu_{6}} & \equiv \int \frac{d^{4} p}{(2 \pi)^{4}} \sum_{\sigma(1,2,4,5,6)} \operatorname{Tr}\left(\gamma^{\mu_{3}} S\left(p+q_{1}+q_{2}+q_{4}+q_{5}+q_{6}\right) \gamma^{\mu_{1}} S\left(p+q_{2}+q_{4}+q_{5}+q_{6}\right)\right. \\
& \left.\times \gamma^{\mu_{2}} S\left(p+q_{4}+q_{5}+q_{6}\right) \gamma^{\mu_{4}} S\left(p+q_{5}+q_{6}\right) \gamma^{\mu_{5}} S\left(p+q_{6}\right) \gamma^{\mu_{6}} S(p)\right) .
\end{aligned}
$$



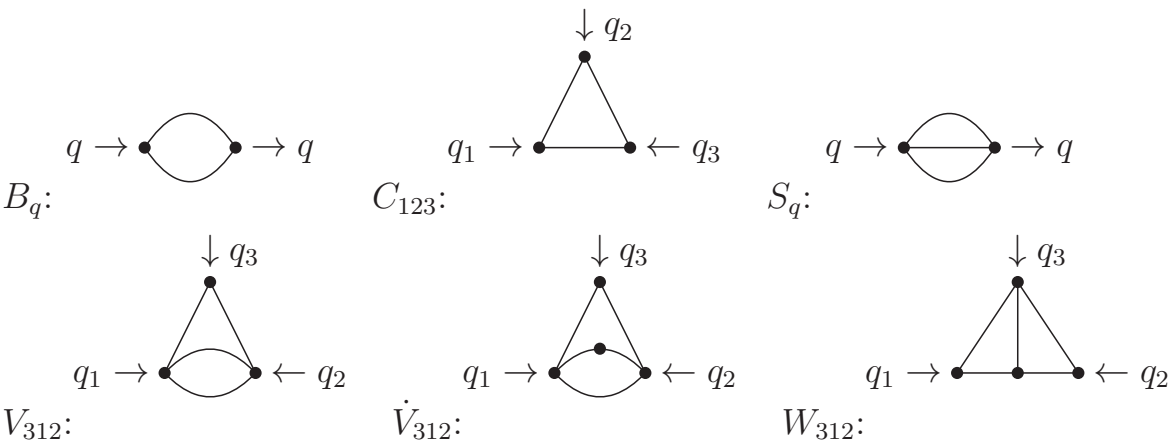

Figure 4. Master integrals entering into the two-loop calculation after applying scalar reduction.

\begin{tabular}{|c|r|r|r|r|r|}
\hline & $\tilde{\Pi}_{1}$ & $\tilde{\Pi}_{7}$ & $\tilde{\Pi}_{10}$ & $\tilde{\Pi}_{13}$ & $\tilde{\Pi}_{19}$ \\
\hline Quark loop & -0.0816 & 0.123 & 0.0363 & 0.0274 & 0.0263 \\
\hline Gluon corrections $\left(\times \pi / \alpha_{s}\right)$ & 0.0781 & -0.136 & -0.0376 & -0.0398 & -0.0411 \\
\hline
\end{tabular}

Table 1. Values for the quark loop and gluonic correction contributions to the $\tilde{\Pi}$ in $\mathrm{GeV}$ units for a benchmark tuple $\left(Q_{1}^{2}, Q_{2}^{2}, Q_{3}^{2}\right)=(1,1.3,1.7) \mathrm{GeV}^{2}$. Sum over the three flavours has been made. The last line is in units of $\alpha_{s} / \pi$.

$S(p)=\frac{p p}{p^{2}}$ is the massless quark propagator and $\sigma(1,2,4,5,6)$ the set of pairwise permutations of $\mu_{i}$ and $q_{i}$ for $i=1,2,3,5,6$.

Now we "simply" need to deal with $\sim 10^{3,4}$ different scalar two-loop integrals, depending on the set of used projectors (which are related by gauge invariance). In order to get the task done we take advantage of KIRA [24], which is a software that allows for a reduction of multi-loop scalar integrals into a set of master integrals. We find that there is a set of them, corresponding to the integrals of Fig 4, for which all the needed $\frac{1}{D-4}$ expansions have been worked out analytically in terms of classical poly-logs [25].

We then have fully analytic results for the five independent scalar functions of the HLbL tensor encoding the g-2 kinematics, which are valid for the regime of large momenta, including the leading term, power and gluonic corrections. We find that gluonic corrections are typically suppressed with respect to the quark loop by a factor of approximately $-\frac{\alpha_{s}}{\pi} .^{2}$ Numerical results for a benchmark point are shown in Table 1.

Now it is a matter of convoluting with the other two loops and numerically integrate gluonic corrections, using Eq. (5), for the kinematics regions where the expansion is valid. This is $Q_{1,2,3}>Q_{\min }$. Having computed the power and gluonic corrections, we can actually assess from which values of $Q_{\min }$ the expansion is safe. The result for the leading massless quark loop and the gluonic corrections is displayed in Fig. 5. We observe how, as far as the running of the strong coupling is well defined, the gluonic corrections are rather small and negative. As a consequence, the obtained expansion gives a very precise description of the (subleading, but not negligible) fully short-distance contributions of HLbL muon g-2.

\footnotetext{
${ }^{2}$ Before realizing of a typo in one of the analytic expressions for the master integrals presented in Ref. [25], the obtained prefactor in front of $\frac{\alpha_{s}}{\pi}$ was $O\left(10^{2,3}\right)$, which would have completely killed the applicability of this expansion.
} 


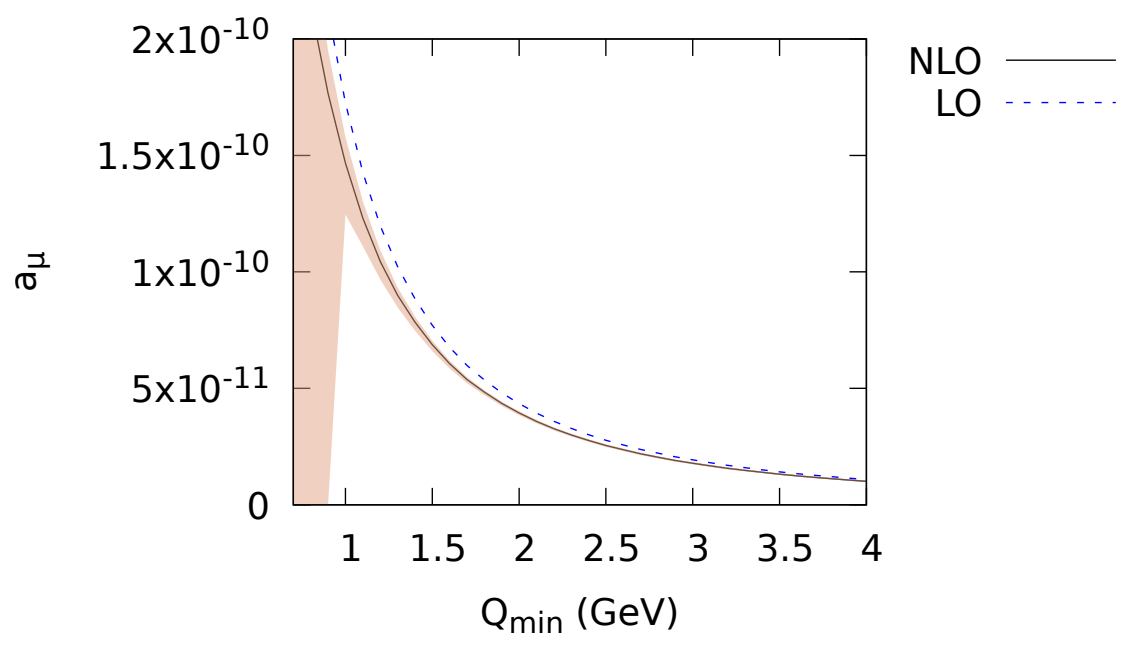

Figure 5. Fully short-distance contributions for HLbL muon g-2, as obtained by our expansion. The leading term (dashed-line) corresponds to the massless quark loop and the NLO one includes the gluonic corrections.

In order to improve the numerical precision, explicit analytic expansions have been performed in some kinematic regions to cancel spurious singularities. One of them corresponds to a sub-region of the Melnikov-Vainshtein short-distance constraints [26]. We have checked how we recover the correct limits for both the quark loop and the gluonic corrections [27, 28].

\section{Conclusions}

We have shown how, in spite of the soft-momentum associated to the external g-2 photon, an OPE can be applied for the HLbL muon g-2 contribution when the three loop momenta are large. The leading contribution is given by the massless quark loop [3].

The leading power correction is found to be suppressed by two powers of the energy, from which one corresponds to the quark mass. In the chiral limit, the first power corrections appear suppressed by four powers of the energy. We have worked out explicit expressions for them in Ref. [4]. They are found to be negligible as far as one is above $\sim 1 \mathrm{GeV}$.

Finally, we have computed the gluonic corrections. The relatively small prefactors in the $\frac{\alpha_{s}}{\pi}$ expansion confirms that it is well behaved from relatively low energies and can be used to improve the precision of the data-driven evaluation of muon $\mathrm{g}-2$.

\section{Acknowledgements}

We thank Laetitia Laub for a fruitful and enjoyable collaboration. This research is supported in part by the Albert Einstein Center for Fundamental Physics at Universität Bern (NHT), the Swedish Research Council grants contract numbers 2016-05996 and 2019-03779 (JB) and the Agence Nationale de la Recherche (ANR) under grant ANR-19-CE31-0012 (project MORA) (ARS). 


\section{References}

[1] B. Abi et al. (Muon g-2), Phys. Rev. Lett. 126, 141801 (2021), 2104.03281

[2] T. Aoyama et al., Phys. Rept. 887, 1 (2020), 2006.04822

[3] J. Bijnens, N. Hermansson-Truedsson, A. Rodríguez-Sánchez, Phys. Lett. B798, 134994 (2019), 1908.03331

[4] J. Bijnens, N. Hermansson-Truedsson, L. Laub, A. Rodríguez-Sánchez, JHEP 10, 203 (2020), 2008. 13487

[5] J. Bijnens, N. Hermansson-Truedsson, L. Laub, A. Rodríguez-Sánchez, JHEP 04, 240 (2021), 2101.09169

[6] G. Colangelo, M. Hoferichter, M. Procura, P. Stoffer, JHEP 09, 074 (2015), 1506.01386

[7] G. Colangelo, M. Hoferichter, M. Procura, P. Stoffer, JHEP 04, 161 (2017), 1702.07347

[8] J. Bijnens, E. Pallante, J. Prades, Phys. Rev. Lett. 75, 1447 (1995), [Erratum: Phys.Rev.Lett. 75, 3781 (1995)], hep-ph/9505251

[9] J. Bijnens, E. Pallante, J. Prades, Nucl. Phys. B 474, 379 (1996), hep-ph/9511388

[10] M. Hayakawa, T. Kinoshita, Phys. Rev. D 57, 465 (1998), [Erratum: Phys.Rev.D 66, 019902 (2002)], hep-ph/9708227

[11] J. Bijnens, E. Pallante, J. Prades, Nucl. Phys. B 626, 410 (2002), hep-ph/0112255

[12] M. Hayakawa, T. Kinoshita (2001), hep-ph/0112102

[13] J. Prades, E. de Rafael, A. Vainshtein, Adv. Ser. Direct. High Energy Phys. 20, 303 (2009), 0901.0306

[14] F. Jegerlehner, The Anomalous Magnetic Moment of the Muon, Vol. 274 (Springer, Cham, 2017)

[15] E.H. Chao, R.J. Hudspith, A. Gérardin, J.R. Green, H.B. Meyer, K. Ottnad, Eur. Phys. J. C 81, 651 (2021), 2104 . 02632

[16] M.A. Shifman, A.I. Vainshtein, V.I. Zakharov, Nucl. Phys. B147, 385 (1979)

[17] M. Jamin (2021), 2106.01614

[18] T. Kadavý, K. Kampf, J. Novotny, JHEP 10, 142 (2020), 2006. 13006

[19] B.L. Ioffe, A.V. Smilga, Nucl. Phys. B232, 109 (1984)

[20] I.I. Balitsky, A.V. Yung, Phys. Lett. 129B, 328 (1983)

[21] A. Czarnecki, W.J. Marciano, A. Vainshtein, Phys. Rev. D67, 073006 (2003), [Erratum: Phys. Rev. D73, 119901 (2006)], hep-ph/0212229

[22] E. Braaten, S. Narison, A. Pich, Nucl. Phys. B373, 581 (1992)

[23] A. Pich, J. Prades, JHEP 06, 013 (1998), hep-ph/9804462

[24] P. Maierhöfer, J. Usovitsch, P. Uwer, Comput. Phys. Commun. 230, 99 (2018), 1705.05610

[25] F. Chavez, C. Duhr, JHEP 11, 114 (2012), 1209. 2722

[26] K. Melnikov, A. Vainshtein, Phys. Rev. D70, 113006 (2004), hep-ph/0312226

[27] J. Lüdtke, M. Procura, Eur. Phys. J. C 80, 1108 (2020), 2006.00007

[28] G. Colangelo, F. Hagelstein, M. Hoferichter, L. Laub, P. Stoffer, Eur. Phys. J. C 81, 702 (2021), 2106. 13222 